\title{
Fractured swab stick as a foreign body in tracheostomy tube
}

\author{
U Hariharan ${ }^{1 *}, N$ Bhasin $^{2}, R$ Sood $^{3}$ \\ Assistant Professor ${ }^{1 *}$, SeniorResident ${ }^{2}$, Head of Department ${ }^{3}$, \\ Department of Anaesthesia and Critical Care, Central Health Services, Govt of India, Dr Ram \\ Manohar Lohia Hospital and PGIMER, New Delhi, India.
}

Corresponding author:uma1708@gmail.com

\begin{abstract}
Tracheostomy is an artificial opening in the trachea in order to provide a clear airway and to remove secretions. Its general indications include upper airway obstruction, prolonged endotracheal intubation, impaired respiratory function, tracheobronchial toilet and to assist in weaning from ventilator. Several complications can be associated with the care of tracheostomy. There have been several case reports of foreign bodies in the tracheostomy tube. We hereby present a unique case of a broken culture swab stick acting as a foreign body inside a tracheostomy tube and hindering effective ventilation in a head injured patient. We also highlight how a change in the tracheostomy tube was required to remove the foreign body from the tracheostomy tube.
\end{abstract}

Keywords: Tracheostomy; foreign body; tube; fractured; swab stick; airway

\section{Introduction}

Tracheostomy is the surgical creation of an artificial opening on the trachea done in the anterior aspect of the neck ${ }^{1}$. The common indications $\mathrm{s}^{2}$ for a tracheostomy include upper airway obstruction, prolonged endotracheal intubation, impaired pulmonary function, tracheobronchial toilet and weaning from ventilator. We hereby present a neurosurgical patient who underwent a surgical tracheostomy following prolonged endotracheal intubation and failure to wean after severe head injury. In view of continued and copious tracheal secretions, a tracheal secretion swab was taken for culture and sensitivity. In the process of taking a culture swab, the distal part of the sterile stick was broken inside the curve of the tracheostomy tube. A change in the tracheostomy tube with full backup for securing the airway was successful in removing the foreign body en-bloc and establishing a patent airway again.

\section{Case Report}

A 35 years old, ASA 1, male sustained a road traffic accident and presented to our emergency department with severe head injury. Patient was intubated due to poor GCS (Glasgow Coma Scale of 7/15). In view of prolonged endotracheal intubation and failure to wean from ventilator, a surgical tracheostomy was done with an $8 \mathrm{~mm}$ cuffed tracheostomy tube and mechanical ventilation continued. Since his tracheal secretions were copious and thick, a decision to collect tracheal secretions for culture and antibiotic sensitivity was taken. Under aseptic precautions, a culture swab was inserted into the tracheostomy tube. During insertion, its distal end inadvertently broke and its fractured fragment was stuck in the tracheostomy tube. The broken segment was placed longitudinally in the lumen of the tracheostomy tube. This placement was recognised immediately and the broken fragment hindered effective ventilation as well as suctioning. Any attempt to retrieve this fragment ran the risk of pushing the foreign body further into the tracheobronchial tree or breaking it into pieces which can lodge anywhere in the tracheobronchial tree. Initially, a paediatric Magill forceps followed by a sterile artery forceps were gently used to grasp the fractured segment, both of which were unsuccessful. The two ends of the fragment were abutting the two side walls of the tracheostomy tube and hence we did not apply any force to retrieve it from above.

After ventilating the patient with $100 \%$ oxygen, the patient was positioned for change of tracheostomy tube. All preparations were made to secure the airway from above, in the event of loss of airway. A standard difficult airway cart including the fibreoptic bronchoscope and all resuscitative equipment were kept ready. An

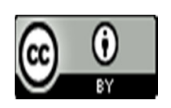

C 2017. Hariharan et al. This is an Open Access article distributed under the terms of the Creative Commons Attribution License (http: //creativecommons.org/licenses/by/4. 0), which permits unrestricted use, distribution, and reproduction in any medium, provided the original work is properly credited 
otorhinolaryngologist was kept standby for any eventuality. A fresh $8 \mathrm{~mm}$ cuffed tracheostomy tube was checked, lubricated and kept ready for insertion, along with one smaller and one larger sized tracheostomy tubes. The patient was adequately sedated and standard monitoring was instituted. The fractured segment was not disturbed and the tracheostomy tube was removed slowly after cuff deflation. The foreign body was removed along with the tracheostomy tube and immediately a fresh $8 \mathrm{~mm}$ cuffed tracheostomy tube was inserted. Air blast from the tracheostomy and bilateral chest air entry were found to be adequate. All vital parameters of the patient were maintained within normal limits. The patient was then connected back to the ventilator. The removed tracheostomy tube was examined for completeness of the fractured segment. (Figures 1 and 2) In order to exclude any retained foreign body in the tracheobronchial tree, a fibreoptic bronchoscopic examination of the airway was immediately done through the new tracheostomy tube after ventilating the patient with $100 \%$ oxygen. The patient remained stable and the entire procedure was uneventful. In due course of time, the patient was weaned from the ventilator and shifted to ward on T-piece with humidified oxygen.

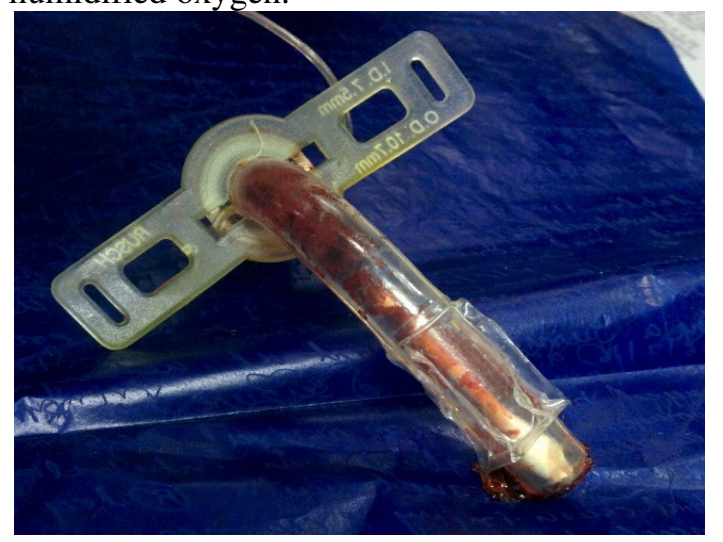

Figure 1

\section{Discussion}

Airway foreign bodies can be catastrophic if not recognised and managed promptly ${ }^{1}$. They can occur in either intubated or non-intubated patients. They are usually accidental and rarely iatrogenic. There have been several case reports of broken fragments of a tracheostomy tube forming airway foreign bodies ${ }^{3}$. Our case is unique as it highlights that iatrogenic airway foreign bodies can be quite difficult to extract.

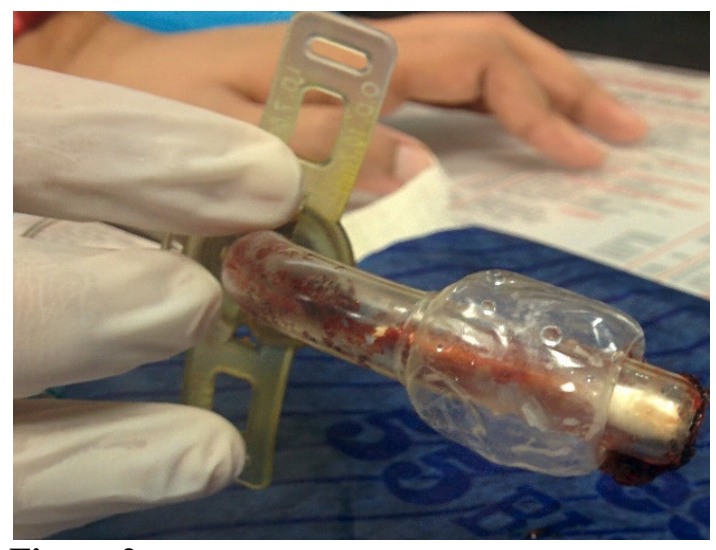

Figure 2

We need to be more careful while taking culture swabs for tracheobronchial secretions. Immediate recognition of this potentially dangerous situation is the first step in management. We had taken all precautions for securing the airway and patient resuscitation before attempting to remove the fractured fragment. A stand by otorhinolaryngologist ${ }^{4}$ was also arranged. We had kept the difficult airway cart $^{5}$ ready, including different sized endotracheal tubes, tracheostomy tubes, fibreoptic bronchoscope, extra oxygen source and other airway equipment.

The position of the broken segment was such that its two ends abutted on the curved inner side walls of the tracheostomy tube. This precarious placement prevented its smooth exit from the tracheostomy tube from above. Even a little force on the fractured segment could have resulted in either further movement downwards or its breakage into pieces. The transverse placement of the broken swab stick in the tracheostomy tube was hindered effective ventilation. The obstructed tracheostomy tube with the foreign body was successfully removed and a fresh tracheostomy tube was inserted. Post-removal, fibreoptic bronchoscopic inspection ${ }^{6}$ and suctioning of the tracheobronchial tree was performed to rule out any residual foreign bodies. Our patient had a reasonable recovery and was finally shifted out after weaning from the ventilator.

\section{Conclusions}

Foreign bodies in the tracheostomy tubes can occur iatrogenically and all efforts must be made to promptly recognize and remove these foreign bodies in the safest way possible. Frequent and

(C) 2017. Hariharan et al. This is an Open Access article distributed under the terms of the Creative 
forceful probing of these foreign bodies must be avoided. All resuscitative equipment, difficult airway cart and a standby otorhinolaryngologist must be available for complete patient safety. Standard ASA monitoring and adequate oxygenation of the patient must be ensured. Bronchoscopic evaluation of the tracheobronchial tree after removal of the foreign body is recommended to rule out any residual fragments.

\section{References}

1. Engels PT, Bagshaw SM, Brindley PG, et al. Tracheostomy: from insertion to decannulation. Can J Surg2009;52(5):427-433.

PMid:19865580 PMCid:PMC2769112

2. Cheung NH, Napolitano LM. Tracheostomy: epidemiology, indications, timing, technique and outcomes. Respir Care 2014;59(6):895-915. https://doi.org/10.4187/respcare.02971 PMid:24891198

3. Swanson KL. Airway foreign bodies: what's new? SeminRespirCrit Care Med 2004;25(4):405-11. https://doi.org/10.1055/s-2004-832713 PMid:16088484

4. Hariharan U. Subglottic stenosis and acute airway obstruction. J Anaesthesiol Clin Pharmacol 2014;30(1):122-124.

https://doi.org/10.4103/0970-9185.125736 PMid:24574619 PMCid:PMC3927282

5. McGuire GP, Wong DT. Airway management: contents of a difficult intubation cart. Can J Anaesth 1999;46(2):190-1.

https://doi.org/10.1007/BF03012557 PMid: 10084003

6. Srppnath J, Mahendrakar V. Management of Tracheobronchial Foreign bodies - A Restrospective Analysis. Indian J Otolaryngol Head Neck Surg 2002;54(2):127-131. 